\title{
Real-World Outcomes of Patients With BRAF-Mutated Metastatic Colorectal Cancer Treated in the United States
}

\author{
Andrew Trunk, $\mathrm{MD}^{1}$; Matthew Braithwaite, $\mathrm{MD}^{1}$; Christopher Nevala-Plagemann, MD²; Lisa Pappas, $\mathrm{MS}^{3}$; \\ Benjamin Haaland, $\mathrm{PhD}^{3}$; and Ignacio Garrido-Laguna, $\mathrm{MD}, \mathrm{PhD}^{2}$
}

\begin{abstract}
Background: BRAF mutations portend a poor prognosis in metastatic colorectal cancer (mCRC). Whether these patients may benefit from more aggressive frontline chemotherapy with a triplet regimen such as FOLFOXIRI remains unclear. We used real-world data from a cohort of patients in the United States to assess the BRAF testing rate, determine the prevalence of FOLFOXIRI use, and compare survival outcomes in $\mathrm{MCRC}$, stratified by BRAF mutation status and firstline therapy. Methods: A nationwide electronic health record-derived deidentified database was reviewed for patients diagnosed with mCRC between 2013 and 2018. Those with documented BRAF mutation testing who received standard first-line therapy were included. Kaplan-Meier estimates with corresponding log-rank tests and Cox proportional hazards modeling compared survival outcomes stratified by BRAF status and first-line therapy. Results: Of 4,457 included patients, 3,991 (89.5\%) had BRAF wild-type (BRAFwt) and $466(10.5 \%)$ had BRAF-mutated (BRAFmt) mCRC. Median overall survival (OS) was 15.4 months in the BRAFmt group versus 28.1 months in the BRAFwt group (hazard ratio $[\mathrm{HR}], 0.48 ; 95 \% \mathrm{Cl}$, $0.41-0.56 ; P<.001)$. Only $3 \%$ of patients with $B R A F$ mutations received first-line FOLFOXIRI \pm bevacizumab, with a median OS of 13.8 months compared with 15.5 months in those treated with doublet chemotherapy \pm bevacizumab $(P=.38)$. In patients with $B R A F$ mutations, propensity-weighted analysis did not detect a significant improvement in OS with FOLFIRI + bevacizumab $(\mathrm{HR}, 0.90 ; 95 \% \mathrm{Cl}$, 0.58-1.39; $P=.63)$ or FOLFOX/CAPEOX + bevacizumab (HR, 0.81; $95 \% \mathrm{Cl}, 0.52-1.26 ; P=.35)$ versus doublet chemotherapy alone. In 2018 , only $56 \%$ of patients diagnosed with $\mathrm{mCRC}$ had documented $B R A F$ testing at any time. Conclusions: This real-world data analysis confirms the negative prognostic impact of BRAF mutations in $\mathrm{mCRC}$ and suggests that FOLFOXIRI has not been widely adopted in the United States. The proportion of patients with documented BRAF testing in this real-world population was low at $56 \%$. We were unable to show any significant difference in OS of patients with BRAFmt $\mathrm{mCRC}$ based on the first-line therapy received.
\end{abstract}

J Natl Compr Canc Netw 2022;20(2):144-150 doi: $10.6004 /$ jnccn.2021.7059

\footnotetext{
${ }^{1}$ Department of Internal Medicine, ${ }^{2}$ Division of Oncology, Department of Internal Medicine, Huntsman Cancer Institute, and ${ }^{3}$ Department of Population Health Sciences, University of Utah, Salt Lake City, Utah.
}

\section{Background}

Colorectal cancer (CRC) remains one of the most common causes of morbidity and mortality in the world and is the second most common cause of cancer death in the United States, with approximately 53,200 deaths annually. ${ }^{1}$ Advances in molecular biology and genomic profiling have led to the identification of several genetic aberrations and tumor characteristics that allow for distinct subgroups of metastatic CRC (mCRC) with both prognostic and predictive implications. One of these subgroups is $B R A F$-mutated (BRAFmt) CRC. BRAF mutations are present in roughly $10 \%$ of patients with $\mathrm{mCRC}^{2}$ and portend a particularly unfavorable prognosis, with a median survival for patients with metastatic disease of $<12$ months. $^{3,4}$ BRAFmt mCRC tumors are typically right-sided, poorly differentiated, and T4 staged, and they characteristically affect older women. ${ }^{5}$

$B R A F$ mutations can be predictive of response to targeted therapy, as shown in the recently published BEACON trial. ${ }^{6}$ In this study, 655 patients with BRAFmt mCRC whose disease progressed on at least one prior line of therapy were randomized to cetuximab + the investigator's choice of either FOLFIRI or irinotecan versus encorafenib (BRAF inhibitor) + cetuximab (anti-endothelial growth factor receptor [EGFR]) or encorafenib + cetuximab + binimetinib (MEK inhibitor). Objective responses were significantly higher and overall survival (OS) was significantly improved in the doublet and triplet arms compared with chemotherapy. ${ }^{6}$ Based on these results, encorafenib gained regulatory approval by the FDA, and the doublet of encorafenib + anti-EGFR therapy has become the second-line standard of care in this patient population.

Optimal first-line (1L) treatment of patients with $B R A F$-positive mCRC remains to be defined. Given the poor prognosis of BRAFmt mCRC, it has been hypothesized that giving FOLFOXIRI up front may give patients the best chance to be exposed to the most active agents

See JNCCN.org for supplemental online content. 
in this disease. Indeed, a phase II trial with FOLFOXIRI in BRAFmt mCRC resulted in a median OS of 24.1 months, which compared favorably with historical control patients. ${ }^{7}$ A randomized phase III study recently evaluated 1L FOLFOXIRI + bevacizumab versus FOLFIRI + bevacizumab in patients with mCRC. ${ }^{8}$ The triplet arm resulted in improved OS (29.9 vs 25.8 months; hazard ratio $[\mathrm{HR}], 0.80 ; 95 \% \mathrm{CI}, 0.65-0.98)$ at the expense of increased toxicity. The survival benefit seemed to be independent of BRAF mutations. However, the study only enrolled a small number of patients with $B R A F$ mutations ( $\mathrm{n}=28)$. Median OS in the BRAF and RAS wildtype (wt) group was 37.1 months compared with 13.4 months for the BRAFmt group (HR, 2.79; 95\% CI, $1.75-4.46 ; P<.0001)$. These results again highlight the poor outcomes associated with BRAFmt mCRC.

Although potential improvement in survival with FOLFOXIRI has been suggested, current NCCN Clinical Practice Guidelines in Oncology (NCCN Guidelines) for Colon Cancer (Version 4.2020) still do not give preference to one specific $1 \mathrm{~L}$ cytotoxic regimen for patients with BRAFmt mCRC. ${ }^{9}$ As such, in this study we sought to use real-world data from a large cohort of patients in the United States to assess changes in BRAF testing rate over time, to determine the prevalence of FOLFOXIRI use, and to compare survival outcomes in mCRC stratified by BRAF mutation status and $1 \mathrm{~L}$ therapy.

\section{Methods}

\section{Data Source}

This study used the nationwide Flatiron Health electronic health record (EHR)-derived deidentified database. During the study period, the deidentified data originated from a geographically diverse group of approximately 280 US cancer clinics ( $~ 800$ centers of care), representing a mixture of academic and community care centers. Deidentified patient-level data in the longitudinal Flatiron Health database include structured data, such as laboratory values, and unstructured data extracted from patient charts using a technology-enabled abstraction process. ${ }^{10,11}$ University of Utah Institutional Review Board approval of the study protocol was obtained before study conduct and included a waiver of informed consent.

\section{Study Population}

We reviewed the EHR-derived deidentified database for patients diagnosed with mCRC between January 1, 2013, and December 31, 2018. Patients who had documented $B R A F$ mutation testing at any time within 6 months of metastatic diagnosis and received standard $1 \mathrm{~L}$ chemotherapy were included for analysis. Standard $1 \mathrm{~L}$ chemotherapy was defined as FOLFOX, CAPEOX, FOLFIRI, or FOLFOXIRI. Patients who did not have a visit or medication order within 90 days of metastatic diagnosis were excluded to ensure that patients were engaged with care at the dataproviding institution.

\section{Statistical Analysis}

Statistical differences between groups were determined using chi-square tests for categorical variables and Wilcoxon rank sum tests for quantitative variables. Kaplan-Meier estimates with corresponding log-rank tests and Cox proportional hazards modeling were used to compare survival outcomes stratified by BRAF mutation status and $1 \mathrm{~L}$ therapy received. Treatment comparative effectiveness was assessed for BRAFmt tumors via a matching weighted Cox proportional hazards model. ${ }^{12,13}$ Matching weights were constructed via treatment propensity scores estimated via a random fores $\mathrm{t}^{14}$ based on age at metastatic diagnosis, sex, metastatic diagnosis year, mismatch repair (MMR) status, ECOG performance status at $1 \mathrm{~L}$ initiation, and de novo versus non-de novo metastatic disease. Proportional hazards assumptions for multivariate Cox proportional hazards models were assessed using Schoenfeld tests and graphical inspection for no pattern in the scaled Schoenfeld residuals across time. An additional multivariate analysis controlled for the same potential confounders. The primary outcome of interest was OS, defined as the time from the date of metastatic diagnosis to the date of death. Statistical analysis was performed using $\mathrm{R}$ version 3.5.2 (R Foundation for Statistical Computing). ${ }^{15}$

\section{Results}

Study Cohort and Patient Characteristics

We selected 20,333 patients diagnosed with mCRC between 2013 and 2018. Within this group, we included 9,948 patients who received standard 1L chemotherapy and had a recorded visit or medication order within 90 days of diagnosis. Of these patients, 4,457 had documented BRAF testing results, with 466 $(10.5 \%)$ listed as having tumors harboring a BRAFmt (Figure 1). Of the $423 B R A F m t$ tumors included in our multivariate analysis, 311 (73.5\%) were V600E, $3(0.7 \%)$ were V600K, 52 (12\%) were "other," and 57 (13\%) were unknown.

Our analysis of the 466 BRAFmt mCRC tumors suggested that patients with these tumors are more likely to be female (55\% vs $42 \%$ for patients with $B R A F \mathrm{wt}$; $P<.0001)$. BRAFmt mCRC tends to present at a later age, with a mean age at metastatic diagnosis of 64 years for $B R A F m$ t versus 60 years for $B R A F \mathrm{wt}(P<.0001)$. We found that $7 \%$ of $B R A F m$ t tumors had concurrent mutations in 


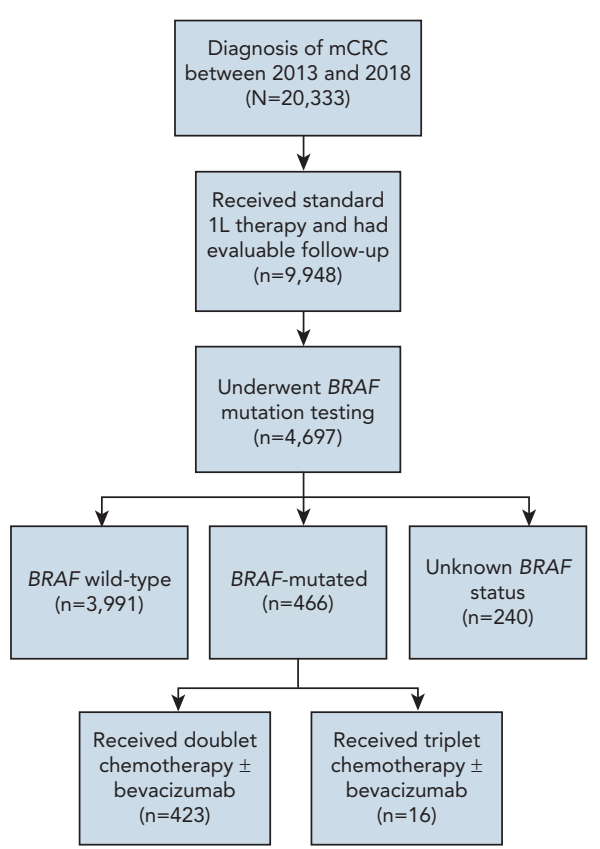

Figure 1. Patient cohort selection flowchart.

Abbreviations: $1 \mathrm{~L}$, first-line; $\mathrm{mCRC}$, metastatic colorectal cancer.

KRAS; $29 \%$ of these $(10 / 34)$ were V600E, and the remaining were either unknown or "other." In patients who were tested for microsatellite instability (MSI)/MMR, we found that $16 \%$ of $B R A F m$ t tumors were MMR-deficient, compared with $3 \%$ of $B R A F$ wt tumors $(P<.0001)$. Demographic and clinical characteristics of the patients included in the study are summarized in Table 1.

\section{Uptake of BRAF Testing}

Testing at any time in the patient disease course for the presence of the BRAF mutation was documented in only $14.1 \%$ of our study patients diagnosed with mCRC in 2013 (Figure 2). Although the proportion of patients with documented $B R A F$ testing increased with each subsequent year, even in the most recent year (2018), only $56 \%$ of patients in this population had documented testing for a $B R A F$ mutation (supplemental eTable 1, available with this article at JNCCN.org). In sum, of all the patients who met our inclusion criteria, only $34.9 \%$ had a documented test for the BRAF mutation.

\section{Prognosis of BRAF Mutations and Uptake of FOLFOXIRI}

The median OS was 15.4 months in the BRAFmt group compared with 28.1 months in the BRAFwt group (Figure 3). A multivariate analysis adjusting for potential confounders, including $1 \mathrm{~L}$ treatment, also showed the negative prognostic implications of a mutation in BRAF (HR, 0.48; 95\% CI, 0.41-0.56; $P<.001$; supplemental eTable 2).
Only $3 \%(\mathrm{n}=16)$ of patients with BRAFmt tumors received 1L FOLFOXIRI, with a median OS of 13.8 months compared with 15.5 months in those receiving a chemotherapy doublet (FOLFOX, CAPEOX, or FOLFIRI; 95\% CI, 4.9-not reached vs 14.3-19.0, respectively; $P=.38$; Figure 4).

In addition, in BRAFmt tumors, matching weighted analysis did not detect a significant improvement in OS with the use of FOLFIRI + bevacizumab (HR, 0.90; 95\% CI, 0.58-1.39; $P=.63$ ) or FOLFOX/CAPEOX + bevacizumab (HR, 0.81; 95\% CI, 0.52-1.26; $P=.35$ ) when compared with a chemotherapy doublet alone. Notably, matching weights achieved well-balanced comparison groups on age at metastatic diagnosis, gender, metastatic diagnosis year, MMR status, ECOG performance status at $1 \mathrm{~L}$ initiation, and de novo versus non-de novo metastatic disease (all standardized mean differences $<0.20$ ). Results based on multivariate analysis were similar (Table 2).

\section{Discussion}

There has been a recent surge of interest in the field of oncology to evaluate the efficacy of various treatment strategies in real-world populations. These analyses can help confirm whether the standard of care as determined in tightly controlled clinical trials is truly applicable to a more general population. This is the largest study evaluating real-world outcomes of $B R A F \mathrm{mt}$ mCRC with frontline therapies. There are several key findings from our study. First, we were able to confirm the negative prognostic implication of a BRAF mutation in mCRC in this large, real-world population. Second, we showed that although the documented $B R A F$ testing rate in this US cohort has increased over time, in 2018 the testing rate still remained quite low at $56 \%$. Third, we showed that the use of up-front triplet chemotherapy has not been widely adopted by oncologists in the United States for treatment of BRAFmt mCRC. Finally, our study did not detect a significant difference in OS between the most commonly used $1 \mathrm{~L}$ treatment strategies for BRAFmt mCRC.

Previous studies have reported the clinicoepidemiologic characteristics of patients with BRAFmt CRC. Our analysis of real-world data is consistent with prior results and shows that patients with $B R A F$ mutations are older, more likely to be female, and more likely to have MMR deficiency compared with patients with $B R A F$ wt. Although we did not subanalyze OS based on $B R A F$ and MSI/MMR status combined, it is known that $B R A F \mathrm{mt} /$ microsatellite stable subgroups tend to fare the worst, and therefore consideration of MSI/MMR status may be a useful additional tool for prognostication in BRAFmt mCRC. ${ }^{16}$ In addition, we were surprised at how many patients had concurrent KRAS and BRAF mutations (7\%). 


\section{Table 1. Patient Characteristics}

\begin{tabular}{|c|c|c|c|}
\hline & $\begin{array}{c}\text { BRAF Mutation } \\
\text { n (\%) }\end{array}$ & $\begin{array}{c}\text { BRAF Wild-Type } \\
n(\%)\end{array}$ & $P$ Value \\
\hline Total, $n$ & 466 & 3,991 & \\
\hline Mean $[\mathrm{SD}]$ age at metastatic diagnosis, y & $64[12]$ & $60[12]$ & $<.0001$ \\
\hline Gender & & & $<.0001$ \\
\hline Female & $257(55)$ & $1,681(42)$ & \\
\hline Male & $209(45)$ & $2,310(58)$ & \\
\hline Asian & $9(2)$ & $90(2)$ & \\
\hline Black or African American & $24(5)$ & $368(10)$ & \\
\hline Hispanic or Latino & $1(0)$ & $12(0)$ & \\
\hline Other & $45(10)$ & $395(11)$ & \\
\hline 2014 & $55(12)$ & $446(11)$ & \\
\hline 2015 & $96(21)$ & $660(17)$ & \\
\hline 2016 & $97(21)$ & $872(22)$ & \\
\hline 2017 & $104(22)$ & $953(24)$ & \\
\hline 2018 & $77(17)$ & 745 (19) & \\
\hline KRAS status & & & $<.0001$ \\
\hline Mutated & $34(7)$ & $1,562(39)$ & \\
\hline Wild-type & $338(73)$ & $1,800(45)$ & \\
\hline Unknown & $94(21)$ & $629(16)$ & \\
\hline 1 & $140(30)$ & $1,162(29)$ & \\
\hline 2 & $41(9)$ & $246(6)$ & \\
\hline $3-4$ & $4(1)$ & $45(1)$ & \\
\hline Missing & $127(27)$ & $1,027(26)$ & \\
\hline Disease presentation & & & .5 \\
\hline De novo metastatic disease & $156(33)$ & $1,277(32)$ & \\
\hline Recurrent disease & $309(66)$ & $2,712(68)$ & \\
\hline Type of standard $1 \mathrm{~L}$ therapy received & & & .13 \\
\hline FOLFOX/CAPEOX/FOLFIRI & $121(26)$ & $1,082(27)$ & \\
\hline FOLFOX/CAPEOX/FOLFIRI + Bev & $302(65)$ & $2,491(62)$ & \\
\hline FOLFOX/CAPEOX/FOLFIRI + anti-EGFR & $27(6)$ & $305(8)$ & \\
\hline FOLFOXIRI & $1(0)$ & $35(1)$ & \\
\hline FOLFOXIRI + Bev & $15(3)$ & $74(2)$ & \\
\hline FOLFOXIRI + anti-EGFR & $0(0)$ & $4(0)$ & \\
\hline
\end{tabular}

Abbreviations: 1L, first-line; Bev, bevacizumab; EGFR, epidermal growth factor receptor; MMR, mismatch repair; MMR-D, mismatch repair deficient; MSI, microsatellite instability; MSI-H, microsatellite instability-high; MSS, microsatellite stable. 




Number at risk

$\begin{array}{rrrrrrr}\text { Untested } & 1,130 & 1,231 & 1,272 & 1,232 & 977 & 627 \\ & 185 & 317 & 541 & 718 & 916 & 802\end{array}$

Figure 2. Documented testing for BRAF mutation within 6 months of metastatic colorectal cancer diagnosis in patients receiving standard first-line treatment between 2013 and 2018.

a May not have 6 months of follow-up in the testing data for those diagnosed in 2018.

Prior literature has also highlighted the poor OS associated with mCRC harboring BRAF mutations. ${ }^{17}$ Our real-world data analysis supports this observation. We found that the median OS of patients with BRAFwt disease was almost double that of patients with $B R A F \mathrm{mt}$ tumors (28.1 vs 15.4 months). Multivariate analysis showed a similar pattern.

The TRIBE study showed numerically improved OS/ progression-free survival in patients with BRAFmt mCRC treated with FOLFOXIRI + bevacizumab versus FOLFIRI + bevacizumab in the first-line setting. Although this finding was not statistically significant, the lower HR in this group compared with the RASwt group suggested a greater mag-

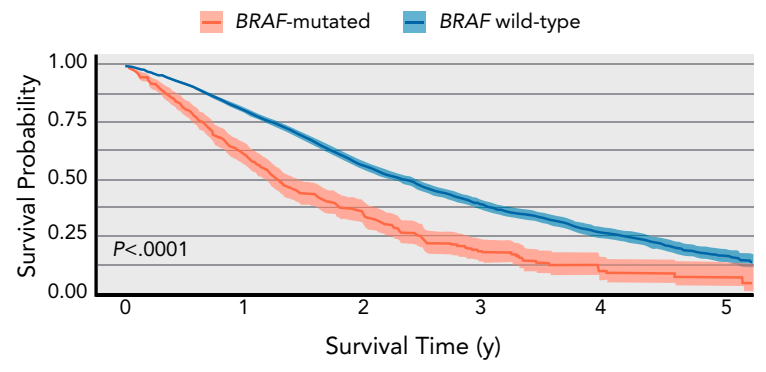

Number at risk BRAF-mutated 466 BRAF wild-type 3,991 224 89 30 9 193 4

Figure 3. Kaplan-Meier curve showing overall survival of patients with metastatic colorectal cancer stratified by BRAF mutational status. nitude of benefit with the triplet in patients with $B R A F$ mutations. ${ }^{8}$ However, the TRIBE2 trial did not confirm the benefit with triplet therapy in patients with $B R A F m t$ mCRC (HR for second progression-free survival [PFS2], 1.23; 95\% CI, 0.72-2.09, with PFS2 representing the time from randomization to objective tumor progression on next-line treatment, or death from any cause). ${ }^{18} \mathrm{~A}$ recent meta-analysis in patients with unresectable mCRC demonstrated imp-rovement in OS with triplet chemotherapy + bevacizumab when compared with doublet chemotherapy + bevacizumab for mCRC in general. ${ }^{19}$ However, when looking specifically at BRAFmt tumors, that same benefit from triplet therapy was not demonstrated. Although only $3 \%$ of the patients in our analysis received $1 \mathrm{~L}$ triplet chemotherapy with FOLFOXIRI + bevacizumab, our real-world data study showed no evidence of a survival benefit for triplet over doublet therapy (13.8 vs 15.5 months; $P=.38$ ).

Although adding antiangiogenics to cytotoxics in patients with $B R A F$ mutations is often done in clinical practice, the role of antiangiogenics in the treatment of BRAFmt tumors remains to be definitively established. ${ }^{20-22}$ Post hoc analysis from studies in both the first- and second-line settings suggests that adding bevacizumab improves survival in patients with $B R A F m t$ tumors. A limitation of this analysis is the small sample size. In the IFL trial, which evaluated the addition of bevacizumab to bolus fluorouracil + irinotecan in the $1 \mathrm{~L}$ setting in $\mathrm{mCRC}$, a trend toward improved survival was seen in the BRAFmt group (15.9 vs 7.9; HR, 0.11; 95\% CI, 0.01-1.06). However, only $10 B R A F m t$ tumors were included in this analysis. The RAISE trial tested ramucirumab (a monoclonal antibody against VEGFR-2) in the second line in combination with FOLFIRI. In the BRAFmt subset $(\mathrm{n}=41)$, there was a nonstatistically significant trend toward improved OS in the ramucirumab arm (9 vs 4.2 months;

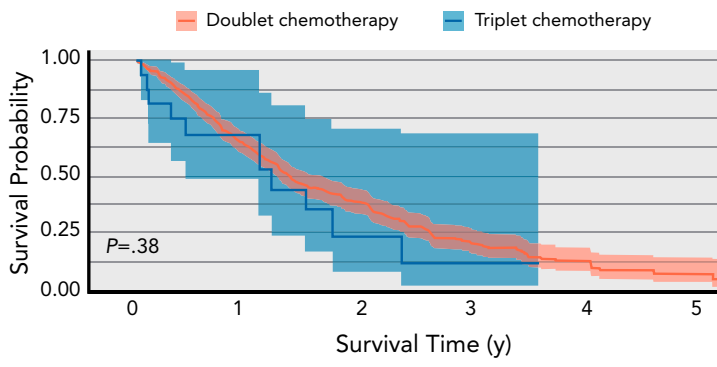

Number at risk Doublet chemotherapy 423

Triplet chemotherapy 16 204 9 82

Figure 4. Kaplan-Meier curve showing overall survival of patients with BRAF mutation receiving doublet chemotherapy (FOLFOX, CAPEOX, or FOLFIRI) versus triplet chemotherapy (FOLFOXIRI). 


\begin{tabular}{|c|c|c|c|}
\hline & n (\%) & HR $(95 \% \mathrm{Cl})$ & $\begin{array}{c}P \\
\text { Value }\end{array}$ \\
\hline Total & 423 & & \\
\hline \multicolumn{4}{|l|}{ Type of $1 \mathrm{~L}$ therapy received } \\
\hline FOLFOX/CAPEOX/FOLFIRI & $121(29)$ & Ref & \\
\hline FOLFIRI + Bev & $68(16)$ & $\begin{array}{c}0.88 \\
(0.50-1.56)\end{array}$ & .666 \\
\hline FOLFOX/CAPEOX + Bev & $234(55)$ & $\begin{array}{c}0.89 \\
(0.59-1.34)\end{array}$ & .577 \\
\hline \multicolumn{4}{|c|}{ Mean [SD] age at metastatic diagnosis } \\
\hline $64.4[12] \mathrm{y}$ & - & $\begin{array}{c}1.00 \\
(0.99-1.02)\end{array}$ & .803 \\
\hline \multicolumn{4}{|l|}{ Gender } \\
\hline Female & $236(56)$ & Ref & \\
\hline Male & $187(44)$ & $\begin{array}{c}0.93 \\
(0.67-1.31)\end{array}$ & .695 \\
\hline \multicolumn{4}{|c|}{ Year of metastatic diagnosis, mean [SD] } \\
\hline $2015.9[1.5]$ & - & $\begin{array}{c}1.16 \\
(1.02-1.33)\end{array}$ & .029 \\
\hline \multicolumn{4}{|l|}{ MMR status } \\
\hline Non-MMR & $207(49)$ & Ref & \\
\hline MMR & $70(16)$ & $\begin{array}{c}1.31 \\
(0.76-2.23)\end{array}$ & .328 \\
\hline Not tested/unknown & $146(34)$ & $\begin{array}{c}0.99 \\
(0.68-1.43)\end{array}$ & .948 \\
\hline \multicolumn{4}{|l|}{ ECOG performance status } \\
\hline 0 & $106(25)$ & Ref & \\
\hline 1 & $105(25)$ & $\begin{array}{c}1.87 \\
(1.29-2.70)\end{array}$ & .001 \\
\hline 2 & $30(7)$ & $\begin{array}{c}2.47 \\
(1.45-4.20)\end{array}$ & .001 \\
\hline $3-4$ & $3(1)$ & $\begin{array}{c}1.95 \\
(0.60-6.34)\end{array}$ & .268 \\
\hline \multicolumn{4}{|l|}{ Metastatic diagnosis } \\
\hline Non-de novo & $142(34)$ & Ref & \\
\hline De novo & $280(66)$ & $\begin{array}{c}1.55 \\
(1.01-2.37)\end{array}$ & .045 \\
\hline
\end{tabular}

Abbreviations: 1L, first-line; Bev, bevacizumab; HR, hazard ratio; MMR, mismatch repair.

HR, 0.54; 95\% CI, 0.25-1.13). The VELOUR trial evaluated the benefit of adding aflibercept (a fusion protein that binds VEGF) to second-line FOLFIRI in patients with mCRC. The study showed a trend toward improved OS in the BRAFmt group $(\mathrm{n}=36)$ that received aflibercept $(10.3$ vs 5.5 months; $95 \% \mathrm{CI}, 0.16-1.09){ }^{23}$ In our analysis (BRAFmt $\mathrm{n}=466$ ), we were not able to show a survival benefit for patients with $B R A F$ mutations who received any doublet therapy (FOLFOX/CAPEOX/FOLFIRI) + bevacizumab compared with those who did not receive bevacizumab. It is possible that the benefit seen in controlled study patients does not translate when this regimen is applied to all-comers with BRAFmt mCRC. However, additional efforts are needed to better understand the role of antiangiogenics in combination with cytotoxics or targeted therapy in BRAFmt tumors.

Of the 9,948 patients included in our study, we were intrigued to note that even in the year of highest testing (2018), only slightly more than half of the patients had a documented test for BRAF mutation (Figure 2). Importantly, of the 5,251 patients (44\%) without documented BRAF testing, only $1,620(30.7 \%)$ had a KRAS or NRAS mutation detected at any time. Thus, using the detection of a $R A S$ mutation as an explanation for the absence of $B R A F$ testing seems to be the case in only a fraction of patients. It is also important to note that this low $B R A F$ testing rate was observed prior to publication of the BEACON trial results in 2019, which showed improved survival with targeted therapy compared with cytotoxics in the second line in V600E BRAF mCRC tumors. ${ }^{6}$ Ideally, these results should drive implementation of universal $B R A F$ testing in this patient population, because encorafenib plus anti-EGFR has been established as a new standard of care in the second line in these patients, especially in the absence of MMR deficiency.

Our study has some limitations. We were able to include only patients who underwent palliative chemotherapy and had standard follow-up and documented $B R A F$ testing. However, the characteristics of our BRAF patient population recapitulate what has been reported in other series and validate our data. The specific $B R A F$ mutation was "unknown" for approximately $25 \%$ of our patient population, which may lead to some dilution of our analysis in patients with non-V600E mutations. In our study, only 16 patients received triplet chemotherapy in the front line, which precludes any definitive conclusion regarding the benefit of triplet therapy in this patient population. In addition, because this is a nonrandomized trial, there is a possibility for selection bias regarding which patients were offered FOLFOXIRI. Concerning $B R A F$ testing, it is possible that there are missing data from patient charts, because the patients may have been tested out of the network our data were drawn from. Consequently, we cannot extrapolate the testing rate to the entire US mCRC population. In addition, because our inclusion was BRAF testing known within 6 months of diagnosis, it is possible that we missed patient testing data from 2018 due to a patient being diagnosed late in the year without mutational testing yet resulted. Final limitations include the retrospective study design and the potential for inclusion bias and unmeasured confounders. 


\section{Conclusions}

In this real-world data analysis, we did not find significant differences in the OS of patients with BRAFmt mCRC based on which type of $1 \mathrm{~L}$ therapy they received. This analysis supports that the presence of BRAFmt portends poor survival outcomes in mCRC. Finally, despite the known negative prognostic impact from BRAF mutations, documented testing rates remain low, with only $56 \%$ of our study patients undergoing testing in 2018. The results of the BEACON trial, published in $2019,{ }^{6}$ are likely to drive an increase in $B R A F$ testing in the future.
Submitted November 26, 2020; final revision received April 17, 2021 accepted for publication May 5, 2021.

Contributions: Project conception: Braithwaite, Nevala-Plagemann, Garrido-Laguna. Data collection and assembly: Braithwaite, NevalaPlagemann, Pappas, Haaland. Data analysis and interpretation: All authors. Manuscript-writing and editing: All authors. Manuscript-approval of final version: All authors.

Disclosures: Dr. Garrido-Laguna has disclosed serving as a principal investigator for Pfizer. The remaining authors have disclosed that they have not received any financial consideration from any person or organization to support the preparation, analysis, results, or discussion of this article.

Correspondence: Ignacio Garrido-Laguna, MD, PhD, Division of Oncology, Department of Internal Medicine, University of Utah School of Medicine, Huntsman Cancer Institute, 2000 Circle of Hope, Suite 5507, Salt Lake City، UT 84112. Email: Ignacio.Garrido-Laguna@hci.utah.edu

\section{References}

1. Siegel RL, Miller KD, Goding Sauer A, et al. Colorectal cancer statistics, 2020. CA Cancer J Clin 2020;70:145-164.

2. Barras D, Missiaglia E, Wirapati $P$, et al. BRAFV600E mutant colorectal cancer subtypes based on gene expression. Clin Cancer Res 2017;23: 104-115.

3. Wang J, Shen J, Huang C, et al. Clinicopathological significance of BRAFV600E mutation in colorectal cancer: an updated meta-analysis. J Cancer 2019:10:2332-2341.

4. Samowitz WS, Sweeney C, Herrick J, et al. Poor survival associated with the BRAF V600E mutation in microsatellite-stable colon cancers. Cancer Res 2005;65:6063-6069

5. Loupakis F, Moretto R, Aprile G, et al. Clinico-pathological nomogram for predicting BRAF mutational status of metastatic colorectal cancer. Br J Cancer 2016;114:30-36.

6. Kopetz S, Grothey A, Yaeger R, et al. Encorafenib, binimetinib, and cetuximab in BRAF V600E-mutated colorectal cancer. N Engl J Med 2019;381:1632-1643.

7. Loupakis F, Cremolini C, Salvatore L, et al. FOLFOXIRI plus bevacizumab as first-line treatment in BRAF mutant metastatic colorectal cancer. Eur J Cancer 2014;50:57-63.

8. Cremolini C, Loupakis F, Antoniotti C, et al. FOLFOXIRI plus bevacizumab versus FOLFIRI plus bevacizumab as first-line treatment of patients with metastatic colorectal cancer: updated overall survival and molecular subgroup analyses of the open-label, phase 3 TRIBE study. Lancet Onco 2015;16:1306-1315.

9. Benson AB III, Venook AP, Al-Hawary MM, et al. NCCN Clinical Practice Guidelines in Oncology: Colon Cancer. Version 4.2020. Accessed April 7 2021. To view the most recent version, visit NCCN.org

10. Ma X, Long L, Moon S, et al. Comparison of population characteristics in real-world clinical oncology databases in the US: Flatiron Health, SEER, and NPCR [preprint published online May 30, 2020]. medRxiv, doi: 10.1101/2020.03.16.20037143

11. Birnbaum B, Nussbaum N, Seidl-Rathkopf K, et al. Model-assisted cohort selection with bias analysis for generating large-scale cohorts from the EHR for oncology research [preprint published online January 13, 2020]. ArXiv, arXiv:2001.09765v 1

12. $\mathrm{Li} \mathrm{L}$, Greene T. A weighting analogue to pair matching in propensity score analysis. Int J Biostat 2013;9:215-234.
13. Yoshida K, Hernández-Díaz S, Solomon DH, et al. Matching weights to simultaneously compare three treatment groups: comparison to threeway matching. Epidemiology 2017;28:387-395.

14. Breiman L. Random forests. Mach Learn 2001;45:5-32.

15. R Core Team. R: A Language and Environment for Statistical Computing. Vienna, Austria: R Foundation for Statistical Computing; 2013.

16. Lochhead P, Kuchiba A, Imamura $Y$, et al. Microsatellite instability and BRAF mutation testing in colorectal cancer prognostication. J Natl Cancer Inst 2013:105:1151-1156.

17. Modest DP, Ricard I, Heinemann V, et al. Outcome according to KRAS-, NRAS- and BRAF-mutation as well as KRAS mutation variants: pooled analysis of five randomized trials in metastatic colorectal cancer by the AIO colorectal cancer study group. Ann Oncol 2016;27:1746-1753.

18. Cremolini C, Antoniotti C, Rossini D, et al. Upfront FOLFOXIRI plus bevacizumab and reintroduction after progression versus mFOLFOX6 plus bevacizumab followed by FOLFIRI plus bevacizumab in the treatment of patients with metastatic colorectal cancer (TRIBE2): a multicentre, open-label, phase 3, randomised, controlled trial. Lancet Oncol 2020;21:497-507.

19. Cremolini C, Antoniotti C, Stein A, et al. Individual patient data metaanalysis of FOLFOXIRI plus bevacizumab versus doublets plus bevacizumab as initial therapy of unresectable metastatic colorectal cancer [published online August 20, 2020]. J Clin Oncol, doi: 10.1200/JCO.20.01225

20. Heinemann $V$, von Weikersthal LF, Decker $T$, et al. FOLFIRI plus cetuximab versus FOLFIRI plus bevacizumab as first-line treatment for patients with metastatic colorectal cancer (FIRE-3): a randomised, open-label, phase 3 trial. Lancet Oncol 2014;15:1065-1075.

21. Hurwitz H, Fehrenbacher L, Novotny W, et al. Bevacizumab plus irinotecan, fluorouracil, and leucovorin for metastatic colorectal cancer. $\mathrm{N}$ Engl J Med 2004;350:2335-2342.

22. Van Cutsem E, Tabernero J, Lakomy R, et al. Addition of aflibercept to fluorouracil, leucovorin, and irinotecan improves survival in a phase III randomized trial in patients with metastatic colorectal cancer previously treated with an oxaliplatin-based regimen. J Clin Oncol 2012;30:3499-3506.

23. Wirapati $P$, Pomella $V$, Vandenbosch $B$, et al. VELOUR trial biomarkers update: impact of RAS, BRAF, and sidedness on aflibercept activity [abstract]. J Clin Oncol 2017;35(Suppl):Abstract 3538. 

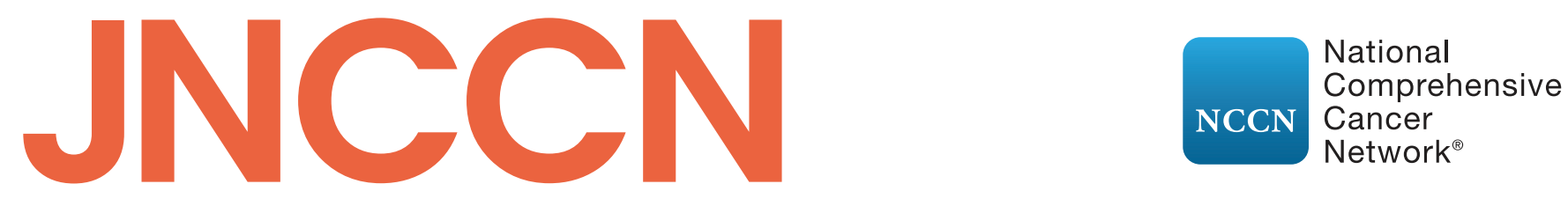

JOURNAL OF THE NATIONAL COMPREHENSIVE CANCER NETWORK

Supplemental online content for:

\section{Real-World Outcomes of Patients With BRAF-Mutated Metastatic Colorectal Cancer Treated in the United States}

Andrew Trunk, MD; Matthew Braithwaite, MD; Christopher Nevala-Plagemann, MD; Lisa Pappas, MS; Benjamin Haaland, PhD; and Ignacio Garrido-Laguna, MD, PhD

J Natl Compr Canc Netw 2022;20(2):144-150

eTable 1: Demographic and Patient Factors at Time of CRC Diagnosis

eTable 2: Multivariate Survival Analysis for Patients With $\mathrm{mCRC}$ and Documented Results From BRAF Mutation Testing 


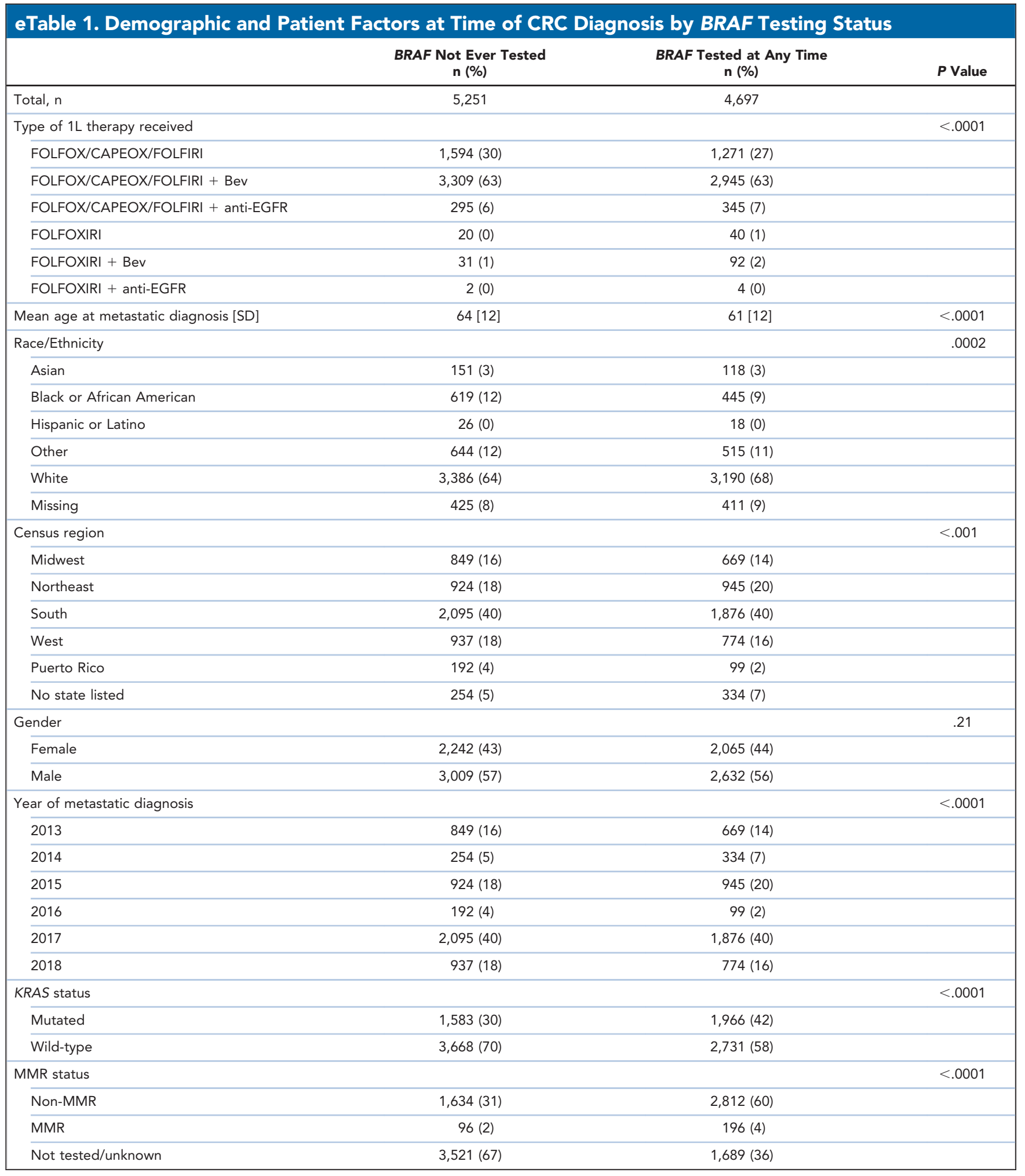


2 - Trunk et al

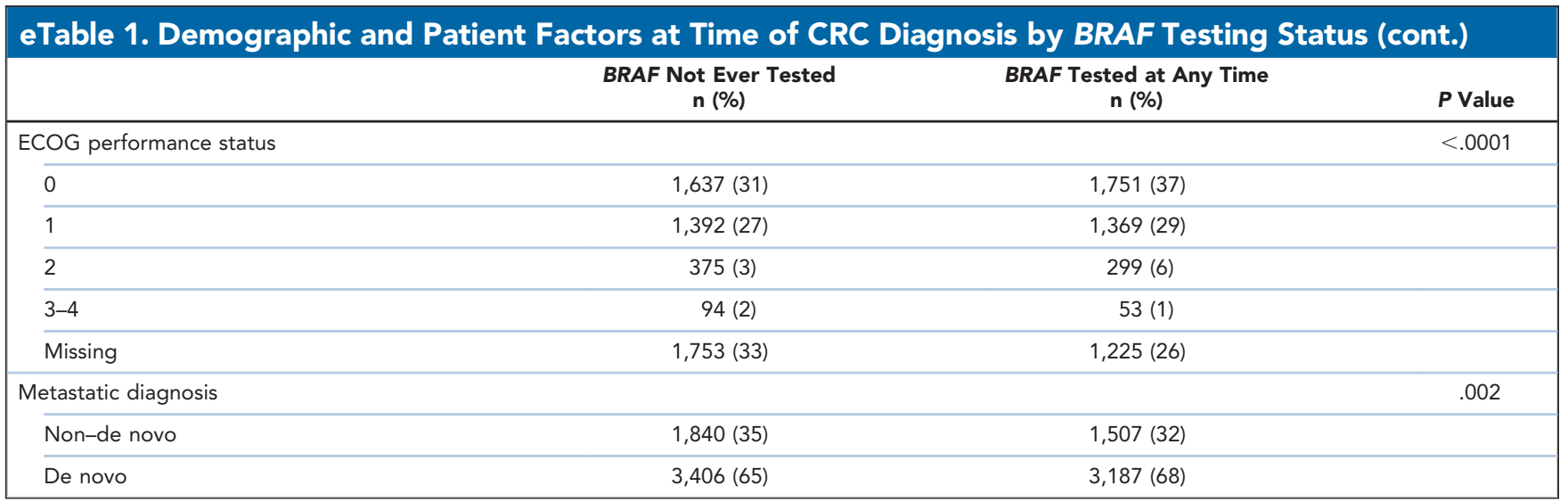

Abbreviations: 1L, first-line; Bev, bevacizumab; CRC, colorectal cancer; EGFR, epidermal growth factor receptor; HR, hazard ratio; MMR, mismatch repair. 


\section{eTable 2. Multivariate Survival Analysis for Patients With $m C R C$ and Documented Results From BRAF} Mutation Testing

\begin{tabular}{|c|c|c|c|}
\hline & n & $\mathrm{HR}(95 \% \mathrm{Cl})$ & $P$ Value \\
\hline Total, $n$ & 4,457 & & \\
\hline \multicolumn{4}{|l|}{ BRAF status } \\
\hline Wild-type & 3,991 & $0.48(0.41-0.56)$ & $<.001$ \\
\hline \multicolumn{4}{|l|}{ Type of $1 \mathrm{~L}$ therapy received } \\
\hline FOLFOX/CAPEOX/FOLFIRI + anti-EGFR & 332 & $0.78(0.60-1.00)$ & .054 \\
\hline FOLFOXIRI & 36 & $0.95(0.47-1.92)$ & .886 \\
\hline FOLFOXIRI + Bev & 89 & $0.67(0.40-1.14)$ & .140 \\
\hline FOLFOXIRI + anti-EGFR & 4 & $4.55(1.13-18.35)$ & .033 \\
\hline Asian & 109 & - & \\
\hline Black or African American & 423 & $1.40(0.95-2.06)$ & .091 \\
\hline Hispanic or Latino & 16 & $2.10(1.00-4.42)$ & .051 \\
\hline Other & 483 & $1.16(0.78-1.71)$ & .467 \\
\hline White & 3,041 & $1.24(0.87-1.76)$ & .242 \\
\hline \multicolumn{4}{|l|}{ Gender } \\
\hline Female & 1,938 & - & \\
\hline Male & 2,519 & $1.09(0.98-1.22)$ & .117 \\
\hline \multicolumn{4}{|l|}{ Year of metastatic diagnosis [SD] } \\
\hline \multicolumn{4}{|l|}{ MMR status } \\
\hline Non-MMR & 2,707 & - & \\
\hline MMR & 187 & $1.09(0.83-1.42)$ & .535 \\
\hline Not tested/unknown & 1,563 & $1.03(0.91-1.16)$ & .660 \\
\hline \multicolumn{4}{|l|}{ ECOG performance status } \\
\hline 0 & 1,665 & - & \\
\hline 1 & 1,302 & $1.48(1.32-1.66)$ & $<.001$ \\
\hline 2 & 287 & $2.11(1.75-2.53)$ & $<.001$ \\
\hline $3-4$ & 49 & $2.59(1.74-3.85)$ & $<.001$ \\
\hline \multicolumn{4}{|l|}{ Metastatic diagnosis } \\
\hline Non-de novo & 1,433 & - & \\
\hline De novo & 3,021 & $1.17(1.05-1.32)$ & .007 \\
\hline
\end{tabular}

Abbreviations: 1L, first-line; Bev, bevacizumab; EGFR, epidermal growth factor receptor; HR, hazard ratio; mCRC, metastatic colorectal cancer; MMR, mismatch repair. 